\title{
Analysis of Some Phytochemicals as Potential Anticancer Agents Targeting the Receptor Protein Associated With Cancer
}

Rishabh Sen ( $\square$ rishabhsen21@gmail.com )

Serampore College Arts Science and Commerce Departments https://orcid.org/0000-0002-3527-0276

Subarna Ghosh

Serampore College Arts Science and Commerce Departments

Anindita Datta

Serampore College Arts Science and Commerce Departments

\section{Research Article}

Keywords: Cancer, Limitations of anticancer drugs, Phytochemicals, Phytocompounds analogs

Posted Date: January 25th, 2022

DOl: https://doi.org/10.21203/rs.3.rs-1246365/v1

License: (c) (i) This work is licensed under a Creative Commons Attribution 4.0 International License.

Read Full License 


\section{Abstract}

Cancerous growth is characterized as the uncontrolled multiplication and spread of the body's particular cells causing infection and addresses one of the greatest medical care issues for humanity and requests a proactive procedure for fix. Sensitization of cancer cells to conventional drugs using multi-target agents that block survival and oncogenic pathways, alone or in combination, is an emerging strategy to overcome drug resistance. Plants are natural reservoirs of novel compounds and provide a promising therapeutic approach for treatment of cancer. Phytochemicals are viewed as appropriate possibility for anticancer medication advancements due to their pleiotropic activities on track occasions with numerous habits. These phytochemicals frequently act by means of regulating molecular pathways which are embroiled in development and dissemination of cancer. The particular strategies in cancer prevention involves tracking of anti-carcinogenic agents, carcinogen inactivation, repressing proliferation, induction of cell cycle capture and apoptosis; and regulating the immune system. Presently, research is in progress for search of novel phytochemicals having anti cancerous properties with minimum or zero side effects. Numerous phytochemicals and their determined analogs have been recognized as possible contender for anticancer treatment and the present review article sheds light on few of these phytocompounds.

\section{Introduction}

Cancer is characterized as the uncontrolled multiplication and spread of the body's particular cells. Cell division is a simple cycle from the earliest starting point of life in the universe. Symmetric cell division prompts multiplication and asymmetric cell division is an enlightening advance for differentiation. It has been accounted that unusual symmetric cell division is the central point for causing cancerous growth. (Shahriyari et al., 2013) According to WHO, 13\% of world deaths accounted in 2005 is a result of cancer. Also, projections have shown that cause-specific years of life lost (YLL) rate because of cancer would go up in 2005, 2015 and 2030. A great many types of plants, animals, marine life forms and microorganisms go about as appealing hotspots for new helpful competitor compounds. In any case, the advancement of novel specialists from regular sources face numerous difficulties that are not normally met when one compares them with engineered compounds. Additionally, there might be challenges with identification, disengagement, evaluating and getting the proper amounts of the dynamic compound in the sample. (WHO, 2016; Rocha et al., 2001) There are numerous pathways that are associated with the cancer development in body. The commitment of plant as medication was disregarded because of the absence of exact biochemical and pharmacological components. Naturally occurring compounds from plants known as phytochemicals, fill in as indispensable assets for novel medications and are likewise rich sources for cancer treatment. The quest for hostile to cancerous growth compounds from plant sources began vigorously during the 1950s with the disclosure and advancement of the different regular mixtures like vinca alkaloids, vinblastine, vincristine and cytotoxic podophyllotoxins. The effective clinical use of disease chemotherapeutic specialists from higher plants for about 50 years has made the scientists more intrigued to direct such kinds of investigations. In the new years, new innovations have been created by 
the researchers to upgrade regular item as drugs to be used in for industrial purposes (Crag et al., 2005; Pan et al., 2010).

These phytochemicals have been tried against cancer viability at both in vitro and in vivo levels. They have complementary mechanisms to hinder the carcinogenic process by searching free radicals, inhibiting survival and multiplication of malignant cells, as well as reducing invasiveness and angiogenesis of tumors. They present wide and complex scope of activities on various molecular targets and signal transduction pathways including receptors, kinases, downstream tumor-activator or suppressor proteins, transcriptional factors, microRNAs (miRNAs), cyclins, and caspases (Choudhary et al., 2020).

\section{Currently Available Drugs For Cancer Treatment And Their Limitations}

Cancer treatment includes physical removal of tumor, radiotherapy and chemotherapy. Treatment strategy relies on the stage and location of tumor. Chemotherapy includes cytotoxic and cytostatic medications and end up being extremely efficient when utilized in blend with different treatments. Legitimate chemotherapeutics include alkylating molecules, topoisomerase inhibitors, tubulin acting agents and antimetabolites. Alkylating agents bind covalently with DNA, crosslink them and produce strand breaks. Carboplatin, cisplatin, oxaliplatin, cyclophosphamide, and melphalan are model alkylating agents which work by causing such damage to DNA. Doxorubicin and irinotecan are topoisomerase inhibitors and block DNA replication. Tubulin acting agents hinder mitotic spindles and arrest mitosis. Paclitaxel, docetaxel, vinblastine and vincristine follow up on tubulins. Paclitaxel (C47H51N014) has been demonstrated as a potent anticancer medication against the vast majority of the cancer types. It was extracted in 1967 from the endophytic fungi found in Taxus brevifolia bark (Carsuso et al., 2000). Paclitaxel targets tubulin and blocks microtubules separation from the centrosomes. The previously mentioned drugs are acceptionally successful against a wide range of cancer, however these medications also have some limitation, during the course of treatment and healthy cells are also targeted and get destroyed like cancerous cells. Sometimes adverse effects of chemotherapy or radiation can lead to drastic changes in the internal cellular environment which might lead to death. There are cells in our body which increase rapidly in number under typical physiological conditions like hair follicle cells, bone marrow cells and digestive tract cells and so forth. The present anticancer medications focus on quick isolation of ordinary cells which is a major challenge, subsequently; give rise to adverse side effects. Because of these results there is decrease in blood formation, GIT aggravation, balding, immunosuppression, heart illnesses and nerve problems may emerge. Another limit is that these cancer cells resist these medications as they go through changes. For instances Drug resistant genes (ABCA4 and ABCA12) were found to be over expressed in human MCF-7 breast cancer cells when docetaxel was applied. In another case, when phytochemical curcumin was applied with docetaxel down regulation of the drug resistant genes (Aung et al., 2017) is observed. Consequently, treating diseased cells by utilizing mono-target chemical agent is certainly not an effective method. In view of wide discoveries, 
phytochemicals and their analogs have most promising choice for better and less toxic cancer treatment (Singh et al., 2016). One of the major impediments in modern chemotherapeutics is multidrug resistance (MDR). The mutation or gene malfunctioning ought to probably result in MDR. Recurrence of chemotherapy can also additionally result in MDR and reduce efflux chemotherapeutic agent outside cell or antiapoptotic mechanism. The MDR proteins reduce the drug efficacy in selected tumor types, showing variable drug penetration rate.

The alternative therapeutic approach became identity of biomarkers earlier than or throughout therapy. The biosensing or diagnostics is an emerging science dealing with identity of biomarkers for MDR tumor types. But time and cost concerned in development are very high (Robey et al, 2018).

The conventional combination therapy wasn't suitable for all malignant MDR tumors either. The conventional therapy did not have selectivity or target ability and fails to provide most appropriate healing response. The selectivity towards most cancers cell is that the major drawback of conventional cancer therapy (Khatoon et al, 2020). Free doxorubicin (DOX, one of the most widely used chemotherapeutic agents for the treatment of cancer) generally has a high resistance factor (RF) value, which is considered to be a significant value.To solve this problem, a kind of silicon nanowire (SiNW)based drug nanocarrier (SiNWDOX) that exhibits high efficiency in the treatment of drug-resistant cancer cells is being reviewed. In general, drug-resistant cancer cells (such as MCF7/ADR cells) are greatly inhibited by SiNW-based nanocarriers, but it is also a very costly and time-consuming step (Peng et al, 2014).

\section{Phytochemicals In Cancer Therapy}

Plants and their bioactive mixtures are in therapeutic practices since long time. Several plant species and their phytochemical derivatives inhibit the development and progress of cancer cells (Aung et al., 2017). It has been explored that plant kingdom comprises roughly 250000 plant species and just around $10 \%$ have been studied for treatment of various diseases. Phytochemicals and their analogues are found in various parts of the plant, e.g., flowers, flower stigma, pericarp, sprouts, organic products, seeds, roots, rhizomes, stem, leaf, bark and play several pharmacological roles. A few plant items like alkaloids, flavonoids, lignans, saponins, terpenes, taxanes, nutrients, minerals, glycosides, gums, oils, biomolecules and other essential and optional metabolites assume critical parts in either repressing disease cell initiating proteins, catalysts and signaling pathways [Cdc2, CDK2 and CDK4 kinases, topoisomerase chemical, cycloxigenase and COX-2 (Cycloxigenase), Bcl-2, cytokines, PI3K, Akt, MAPK/ERK, MMP, TNK,mechanistic target of rapamycin (mTOR) (Figure 1) or by enacting DNA repair system (p21, p27, p51, p53 qualities and their protein items), Bax, Bid, Bak proteins stimulates the development of protective enzymes (Caspase-3, 7, 8, 9, 10, 12), instigating cancer prevention activity (antioxidant enzymes for example GSH, GST and GPxn), hence showing strong anticancer impacts

as far as their viability on the previously mentioned proteins, chemicals and signaling pathways are concerned (Figure 2) (Thakore et al., 2012; Tariq et al., 2017). 


\section{Molecular Targets And Mechanism Of Action Of Anticancer Phytochemicals}

A phytomolecule can inhibit the development of pre- neoplastic cell carcinomas, and interfere with the metabolic transformation of pro-carcinogen. Likewise, they can regulate cell and signaling events engaged with development, invasion and metastasis of cancer cell.

Flavanones, isoflavones and lignans restrict the estrogen to the cancer cells and reduce their multiplication. Decrease of inflammation processes through concealment of nuclear factor-kappa B (NFkB) family transcription factors is a separate mechanism. Curcumin, bilberries anthocyanins, EGCG, caffeic

acid and derivatives and quercetin act through NF-kB signalling pathway.

Luteolin hinders epithelial mesenchymal transition. Other than these mechanisms, anticancer phytomolecules focuses on a few other signalling molecules or pathways additionally to diminish the development and metastasis of cancer cells (lqbal et al., 2017).

Apigenin, a flavone contained in parsley, celery and chamomile, promotes the apoptosis of lung adenocarcinoma cells by targeting the leptin/leptin receptor pathway. It induces caspase-dependent extrinsic apoptosis in human epidermal growth factor receptor 2 (HER2) through the expression of BT474 breast cancer cells by restriction of the signal transducer and stimulant. STAT3 (Seo et al., 2015). It was revealed that apigenin inhibits the PI3K / AKT / mTOR pathway and induces apoptosis and autophagy in hepatic carcinoma cells (Yang et al, 2018).

Curcumin, the polyphenol of Curcuma longa hinders the development of human glioblastoma cells by modulating several molecular segments. It up regulates p21, p16, p53, early growth response protein 1 (Egr-1), extracellular signal regulated kinase (Erk), c-Jun-N-terminal kinase (JNK), ElK-1, Bcl-2 associated X protein (Bax) and Caspase-3, 8, 9 proteins; and down regulates the degrees of B-cell lymphoma 2 (Bcl-2), mechanistic target of rapamycin (mTOR), p65, B-cell lymphoma extra-enormous protein (Bcl-xL), protein kinase B(Akt), epidermal growth factor receptor (EGFR), NF-kB, cell division cycle protein 2 (cdc-2), retinoblastoma protein (pRB), cyclin-D1 proteins and cell myelocytomatosis oncogenes(c-myc) (Vallianou et al., 2015).

Crocetin, the carotenoid present in Crocus sativus and Gardenia jasminoides follow up on GATA binding protein 4 and MEK-ERK1/2 pathway and prevents cardiac hypertrophy.

Cyanidin glycosides from red berries execute antioxidants and anticancer capacities through different process. In colon cancer cells, they smother the statements of inducible nitric oxide synthase (iNOS) and cyclooxygenase-2 (COX-2) genes and hinder mitogen induced metabolic pathways (Cai et al., 2009; Serra et al., 2013). 
EGCG, the catechin polyphenol of tea shows anticancer effects through different mechanisms. It blocks NF-kB initiation, Bcl-2 and COX-2 expresion in prostate carcinoma cells and induces apoptosis. In bladder and lung carcinoma cells, it restrains matrix metallopeptidase-9 (MMP-9) expression. In head and neck carcinoma cells it suppresses the development of vascular endothelial development factor (VEGF). In fibrosarcoma cells it suppresses the Erk phosphorylation and action of MMP-2 and 9. Furthermore, in gastric carcinoma cells it blocks the Erk, JNK, and MMP-9 expression (Singh et al., 2011).

Fisetin and hesperetin cause cell cycle capture in human acute promyelocytic leukemia HL-60 cells by modifying a few signaling pathways, viz. mitogen initiated protein kinases (MAPK), NF-kB, JAK/STAT, $\mathrm{PI3K} / A k t$, Wnt, and mTOR pathways. It shows double inhibition of PI3K/Akt signaling pathways in the lungs cancer (Adan et al., 2015).

Genistein, the isoflavone of soybean shows its anticancer impacts through inhibition of NF-kB and Akt signaling pathways and Gingerol focuses on the Erk1/2/JNK/AP-1 signaling and promotes caspasedependent apoptosis in colon cancer (Radhakrishnan et al., 2014; Joo et al., 2016).

Lycopene targets the PI3K/Akt pathway in pancreatic cancer. It blocks gastric carcinogenesis by hindering Erk and Bcl-2 signaling. It initiates cell antioxidant enzymes (e.g., GST, GSH, and GPx) in the cancerous cells and eliminates oxidative malfunctions created by the carcinogens (Kim et al., 2015).

Rosmarinic acid decreases the activity of COX-2 and Erk phosphorylation in colon cancer cells. In breast cancer, rosmarinic acid lessens the action of DNA methyl transferase and interfere RANKL/RANK/OPG networks. Additionally, it targets PKA/CREB/MITF pathway and NF-kB enactment in melanoma and leukemia U938 cells (Hossan et al., 2014).

Calcitriol restrains prostaglandins, COX-2, NF-kB, and VEGF signalling and prevents angiogenesis of cancer cells. Tocotrienols and g-tocopherol prevent PI3K/Akt and Erk/MAPK pathways (Sylvester et al., 2013).

Colchicine upregulates dual specificity phosphatase 1 (DUSP1) quality in gastric cancer. It also suppresses the development of hepatocellular carcinoma cells through upregulation of A-kinase anchoring protein 12(AKAP12) and transforming growth factor beta-2(TGF-b2) proteins (Kuo et al., 2015).

Podophyllotoxin blocks the development of MCF-7 breast cancer cells by modifying checkpoint kinase 2 (Chk-2) signaling pathway. Podophyllotoxin likewise advances apoptosis in lung carcinoma cells through ER stress, autophagy and cell cycle capture (Choi et al., 2012).

Oroxylin A may be a conceivable isoflavone extracted from Scutellariae radix that down-regulates COX2 and iNOS gene expression, blocks NFkB, and suppresses LPS-induced NFkB activation. Oroxylin A with 5FU is additionally accustomed treat colorectal cancers, showing twofold activity with COX-2 reduction and expanded ROS production (lqbal et al., 2017). 
Taxanes show promising anticancer properties that act by binding to microtubules and has key part in cellular division. Paclitaxel (taxol) was first removed from the bark and leaf of Taxus baccata and $T$. canadensis, Corylus avellana and is used to mend a large scope of cancer growths including ovarian, breast and therefore the lungs. Binding of paclitaxel with $\beta$-tubulin within the lumen of microtubules prompts decline in microtubule dynamics and stop cell cycle at $\mathrm{M}$ stage while docetaxel, a semi synthetic subsidiary from $T$. baccata is basically utilized in breast, pancreas, prostate and lung cancer treatments. (lqbal et al., 2017)

Resveratrol is a naturally occurring polyphenol and has been distinguished in mulberries, peanuts, grapes, bilberries and blueberries. Resveratrol plays important part in relieving a large scope of cancer including breast, colorectal, liver, pancreatic, prostate and lung carcinoma by up-regulating p53 and Bcl-2 related $\mathrm{X}$ proteins and down-regulating NF-kB, MMPs, Bcl-2, A P-1, cyclins, cyclin dependent kinases, cytokines, and COX-2 proteins. Resveratrol is thought to hinder angiogenesis, suppressing VEGF protein activity by decreasing MAP kinase phosphorylation (Patel et al., 2010).

Chrysin [5,7-dihydroxyflavone], is a successful anticancer compound showed less adverse effects and have solid antitumor consequences for various cancer growth cell lines (DU145 and PC-3). This flavone has also initiated apoptosis in SW480 colorectal disease, capture cell cycle at G2/M stage, brings about DNA cleavage and apoptosis, expanding ROS production and lipid peroxidation, suppresses the S6, AKT, $\mathrm{PI} 3 \mathrm{~K}, \mathrm{P} 90 \mathrm{RSK}$ and P70S6K, proteins, induces MAPK and ERK1/2 and P38 proteins within the prostate cancer cells (lqbal, et al., 2017).

Chyricin induces prostate cancer cell death by inducing reactive oxygen species (ROS) and endoplasmic reticulum stress through inhibition of PI3K/AKT signaling (Ryu et al., 2017). Chrysin has also been shown to reinforce the sensitivity of tumour cells to doxorubicin by suppressing the cascade of PI3K/AKT (Issinger et al., 2021).

$\beta$-elemene a sesquiterpene, is moreover a promising anticancer expert with a large extent of its effect against drug resistant tumors and has been extracted from Curcuma wenyujin. $\beta$-elemene controls different kinds of cancer, advances apoptosis and cell death, hinder the expression of VEGF, downregulates Akt phosphorylation and CD34 suppression, hindering PI3K/Akt/mTOR, MAPK and pathway, tightening angiogenesis and upregulates the $\mathrm{Cbl-b}$ and c-Cbl and E3 ubiquitin ligases in human gastric cancer (lqbal et al., 2017).

Thymoquinone (2-isopropyl-5-methyl-1, 4-benzo-quinone, TQ) is the strong constituent of black cumin (Nigella sativa) seed oil. In a BALB/c a thymic naked mice, thymoquinone decreased tumor weight and size by advancing apoptosis and subduing STAT3 phosphorylation in human gastric cancer cells. The downregulation of STAT3 activation in relationship with a decrease in JAK2 and c-Src activity. In another examination in BALB/c mice migrated with mouse epithelia breast cancer EMT6/P cell line, TQ and melatonin combination fundamentally reduced the tumor size, induced tumor cell death, lessened VEGF expression, and enacted anticancer immune response by extending INF-y level (Zhao et al., 2016). 
Ursolic acid (UA) is a natural terpene compound found in number of plants. The in vitro assessment suggested that treatment of CRC cells with UA and oxaliplatin essentially decreased cell duplication, extended apoptosis and ROS development, and prevented the expression of drug resistant gene (Zhang et al., 2018). The UA nanoparticles lessened tumor size by focusing on caspases and p53 with downregulation of Bcl-2 and clAP, starting apoptosis and inciting cervical cancer cell demise (Wang et al., 2018).

These phytomolecules follow up on epigenetic components also. DNA methylation, histone modifications and miRNA expression are significant epigenetic measures which include in cancer growth initiation and growth. Phytomolecules decrease the functions and expressions of DNA methyl transferases (DNMTs), histone deacetylases (HDACs) and histone methyl transferases (HMTs) and increases promoter demethylation in different cancer models (Thakur et al., 2014).

\section{Future Perspectives And Concluding Remarks}

It has been observed that phytochemicals fill in as promising and compelling exploration region with splendid future in cancer treatment. The developing instances of disease and significant expense, different constraints in the traditional treatment including significant expense, and adverse side effects of present anticancer medications has confronted a serious test to every researcher to plan an alternative source, which is eco-friendly, biocompatible and cost-effective technique in a greener way. Under this situation, phytomolecules are relied upon to change the traditional cancer treatment in the following decade. Bioactive phytochemicals are expected leads for the advancement of more secure anticancer medications. High bio degradability and biocompatibility have expanded the viability of these phytomolecules in cancer treatment. A few plants and their constitutive phytochemicals have been evaluated for this reason but not many have reached up to the clinical level. They should be created as druggable structures for cancer with adequate bioavailability. This thorough review paper gives data on medicinal plants and their bioactive compounds with the potential to cure various sorts of cancer growth.

\section{Declarations}

Funding

Not applicable.

\section{Competing Interest}

No conflict of interest among the authors.

\section{Author Contributions}


All authors contributed to the study conception and design. The idea and topic were suggested by Dr.Subarna Ghosh. Literature search and data review were performed by Anindita Datta and the manuscript was critically revised and put together by Rishabh Sen. All authors read and approved the final manuscript.

\section{Ethics Approval}

Not applicable.

\section{Consent to participate}

Authors give their full consent to participate.

\section{Consent to publish}

Authors give their full consent to publish this article.

\section{References}

1. Adan, A., \& Baran, Y. (2015). The pleiotropic effects of fisetin and hesperetin on human acute promyelocytic leukemia cells are mediated through apoptosis, cell cycle arrest, and alterations in signaling networks

2. Aung, T. N., Qu, Z., Kortschak, R. D., \& Adelson, D. L. (2017). Understanding the effectiveness of natural compound mixtures in cancer through their molecular mode of action. Int J Mol Sci, 18(3), 656

3. Carsuso, M., Colombo, A. L., Fedeli, A. L., Pavesi, A., Quaroni, S., Saracchi, M., et al. (2000). Isolation of endophytic fungi and actinomycetes taxane producers. Ann.Microbiol, 50, 3-13

4. Cai, J., Yi, F. F., Bian, Z. Y., Shen, D. F., Yang, L., Yan, L., et al. (2009). Crocetin Protects against cardiac hypertrophy by blocking MEK-ERK1/2 signaling pathway. J. Cell. Mol. Med, 13, 909-925. doi:10.1111/j.1582-4934.2008.00620.x

5. Chen, W., Lu, Y., Wu, J., Gao, M., Wang, A., \& Xu, B. (2011). Beta-elemene inhibits melanoma growth and metastasis via suppressing vascular endothelial growth factor-mediated angiogenesis. Cancer Chemother Pharmacol, 67, 799-808. doi: 10.1007/s00280-010-1378-x

6. Choi, J. Y., Hong, W. J., Cho, J. H., Kim, E. M., Kim, J., Jung, C. H., et al. (2015). Podophyllotoxin acetate triggers anticancer effects against non-small cell lung cancer cells by promoting cell death via cell cycle arrest, ER stress and autophagy

7. Choudhari, S. A., Mandave, C. P., Deshpande, M., Ranjekar, P., \& Prakash, O. (2020). Phytochemicals in Cancer Treatment: From Preclinical Studies to Clinical Practice. Front. Pharmacol. 
doi.org/10.3389/fphar.2019.01614

8. Cragg, G., \& Newman, M., D., J (2005). Plants as a source of anti-cancer agents. J. Ethnopharmacol, 100(1-2), 72-79

9. Da, R., Lopes, A. B., R.M. and, \& Schwartsmann, G. (2001). Natural products in anticancer therapy. Curr. Opin. Pharmacol, 1(4), 364-369

10. Khatoon, E., Banik, K., Harsha, C., Sailo, B. L., Thakur, K. K., Khwairakpam, A. D., et al. (2020).

Phytochemicals in cancer cell chemosensitization: current knowledge and future perspectives. Semin Cancer Bio/10.1016/j.semcancer. 2020.06.014

11. Hossan, M. S., Rahman, S., Bashar, A. B. M. A., Jahan, R., Al-Nahain, A., \& Rahamatullah, M. (2014). Rosmarinic acid: Are view of its anticancer action. WorldJ.Pharm.Pharm.Sci, 3, 57-70

12. Iqbal, J., et al. (2017). Plant-derived anticancer agents: A green anticancer approach. Asian Pac J Trop Biomed. https://doi.org/10.1016/j.apjtb.2017.10.016

13. Issinger, O., \& Guerra, B. (2021). Phytochemicals in cancer and their effect on the PI3K/AKT-mediated cellular signalling. Biomedicine and Pharmacotherapy, 139, 0753-3322.

https://doi.org/10.1016/j.biopha.2021.111650.

14. Joo, J. H., Hong, S. S., Cho, Y. R., \& Seo, D. W. (2016). 10-Gingerol inhibits proliferation and invasion of MDA-MB-231 breast cancer cells through suppression of Akt and p38MAPK activity. Oncol. Rep, 35(2), 779-784. doi: 10.3892/or.2015.4405

15. Kim, M. J., \& Kim, H. (2015). Anticancer effect of lycopene in gastric carcinogenesis. J.CancerPrev, 20, 92-96. doi:10.15430/JCP.2015.20.2.92

16. Koh, C. Y., Ho, T. C., \& Pan, H. M. (2020). O Recent advances in cancer chemoprevention with phytochemicals Journal of Food and Drug Analysis. 28, Issue 1, Pages 16

17. Kuo, M. C., Chang, S. J., \& Hsieh, M. C. (2015). Colchicine significantly reduces incident cancer in Gout male patients

18. Shahriyari, L., \& Komarova, N. L. (2013). “Symmetric vs. asymmetric stem cell divisions: an adaptation against cancer?,"PloS One, vol. 8, Article ID e76195.

19. Pan, L., Chai, H., \& Kinghorn, A., D (2010). The continuing search for antitumor agents from higher plants. Phytochem. Lett, 3(1), 1-8

20. Patel, K. R., Brown, V. A., Jones, D. J., Britton, R. G., Hemingway, D., Miller, A. S., et al. (2010). Clinical pharmacology of resveratrol and its metabolites in colorectal cancer patients. Canc Res, 70, 73927399

21. Peng, F., Su, Y., Ji, X., Zhong, Y., Wei, X., \& He, Y. (2014). Doxorubicin-loaded silicon nanowires for the treatment of drug-resistant cancer cells. Biomaterials.

https://doi.org/10.1016/j.biomaterials.2014.03.032.

22. Radhakrishnan, E. K., Bava, S. V., Narayanan, S. S., Nath, L. R., Thulasidasan, A. K. T., Soniya, E. V., et al. (2014). 6-Gingerol induces caspase dependent apoptosis and prevents PMA-induced proliferation in colon cancer cells by inhibiting MAPK/AP-1 signaling 
23. Robey, R. W., Pluchino, K. M., Hall, M. D., Fojo, A. T., Bates, S. E., \& Gottesman, M. M. (2018). Revisiting the role of $A B C$ transporters in multidrug-resistant cancerNat Rev Cancer.18 (7), pp.452-464

24. Seo, H. S., Jo, J. K., Ku, J. M., Choi, H. S., Choi, Y. K., et al. (2015). Induction of caspase dependent extrinsic apoptosis through inhibition of signal transducer and activator of transcription 3 (STAT3) signaling in HER2-overexpressing BT-474 breast cancer cells. Biosci.Rep, 35, e00276.doi:10.1042/BSR20150165

25. Serra, D., Paixao, J., Nunes, C., Dinis, T. C. P., \& Almeida, L. M. (2013). Cyanidine-3-Glucoside suppresses cytokine induced inflammatory response anticancer Phytochemicals in human intestinal cells: Comparison with 5-aminosalicylic acid. PLOSONE, 8, e73001. doi:10.1371/journal.pone.0073001

26. Singh, B. N., Shankar, S., \& Srivastava, R. K. (2011). Green tea catechin, epigallocatechin-3gallate(EGCG): mechanisms, perspectives and clinical applications. Biochem.Pharmacol, 82, 18071821. doi: 10.1016/j.bcp.2011.07.093

27. Singh, S., Bhupender, S., Kanwar, S. S., \& Kumar, A. (2016). Lead phytochemicals for anticancer drug development. Front Plant Sci, 7, 1667

28. Sylvester, P. W., \& Ayoub, N. M. (2013). Tocotrienols target PI3K/Akt signaling in anti-breast cancer therapy

29. Tariq, A., Sadia, S., Pan, K., Ullah, I., Mussarat, S., Sun, F., et al. (2017). A systematic review on ethnomedicines of anti-cancer plants. Phytother Res, 31, 202-264

30. Thakore, P., Mani, R. K., \& Kavitha, S. J. (2012). A brief review of plants having anti-cancer property. Int J Pharm Res Dev, 3, 129-136

31. Thakur, V. S., Deb, G., Babcook, M. A., \& Gupta, S. (2014). Plant phytochemicals as epigenetic modulators: role in cancer chemoprevention. AAPSJ, 16, 151-163. doi:10.1208/s12248-013-9548-5

32. Vallianou, N. G., Evangelopoulos, A., Schizas, N., \& Kazazis, C. (2015).Potential anticancer properties and mechanisms of action of curcumin

33. World health statistics (2006).1-80, WHO (http://www.who.int/)

34. Yang, C., Pi, C., \& Wang, G. (2018). Inhibition of PI3K/Akt/mTOR pathway by apigenin induces apoptosis and autophagy in hepatocellular carcinoma cells. Biomed Pharm, 103, 699-707

35. Zhang, W., Su, J., Xu, H., Yu, S., Liu, Y., Zhang, Y., et al. (2017b). Dicumarol inhibits PDK1 and targets multiple malignant behaviors of ovarian cancer cells. PloS One, 12(6), e0179672. doi:

10.1371/journal.pone.0179672

36. Zhao, G., Han, X., Zheng, S., Li, Z., Sha, Y., Ni, J., et al. (2016). Curcumin induces autophagy, inhibits proliferation and invasion by downregulating AKT/mTOR signaling pathway in human melanoma cells. Oncol. Rep, 35(2), 1065-1074. doi: 10.3892/or.2015.4413

37. Zhu, W. Q., Wang, J., Guo, X. F., Liu, Z., \& Dong, W. G. (2016). Thymoquinone inhibits proliferation in gastric cancer via the STAT3 pathway in vivo and in vitro. World J. Gastroenterol, 22(16), 41494159. doi: 10.3748/wjg.v22.i16.4149. 


\section{Table}

Due to technical limitations, table 1 is only available as a download in the Supplemental Files section.

Figures

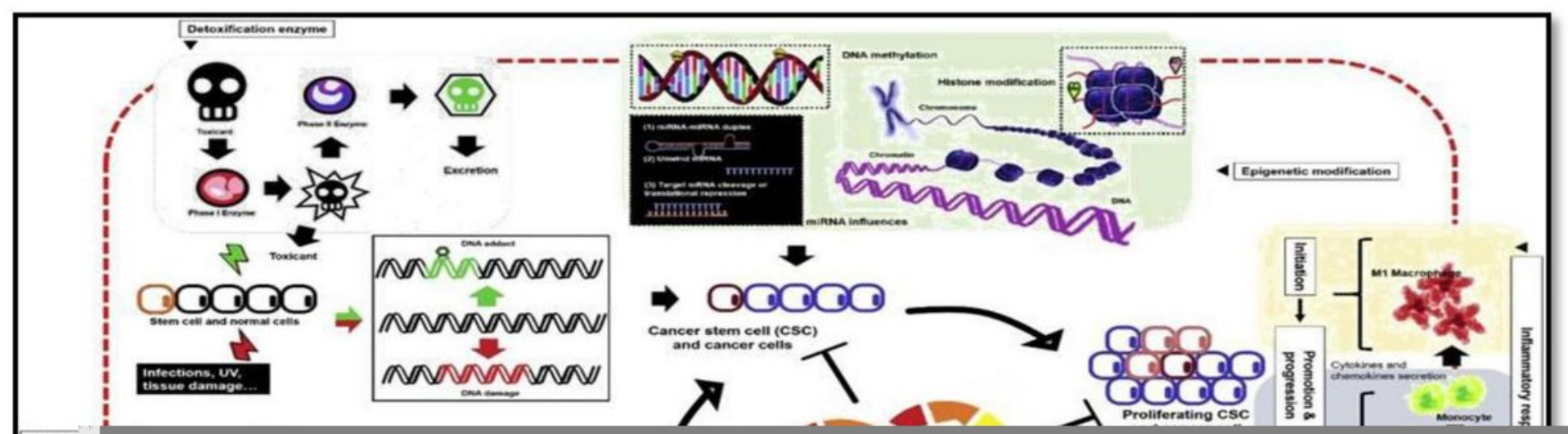

Figure 1

Cancer development and strategy of cancer therapy with the intervention of phytochemicals (after Koh et al., 2020) 


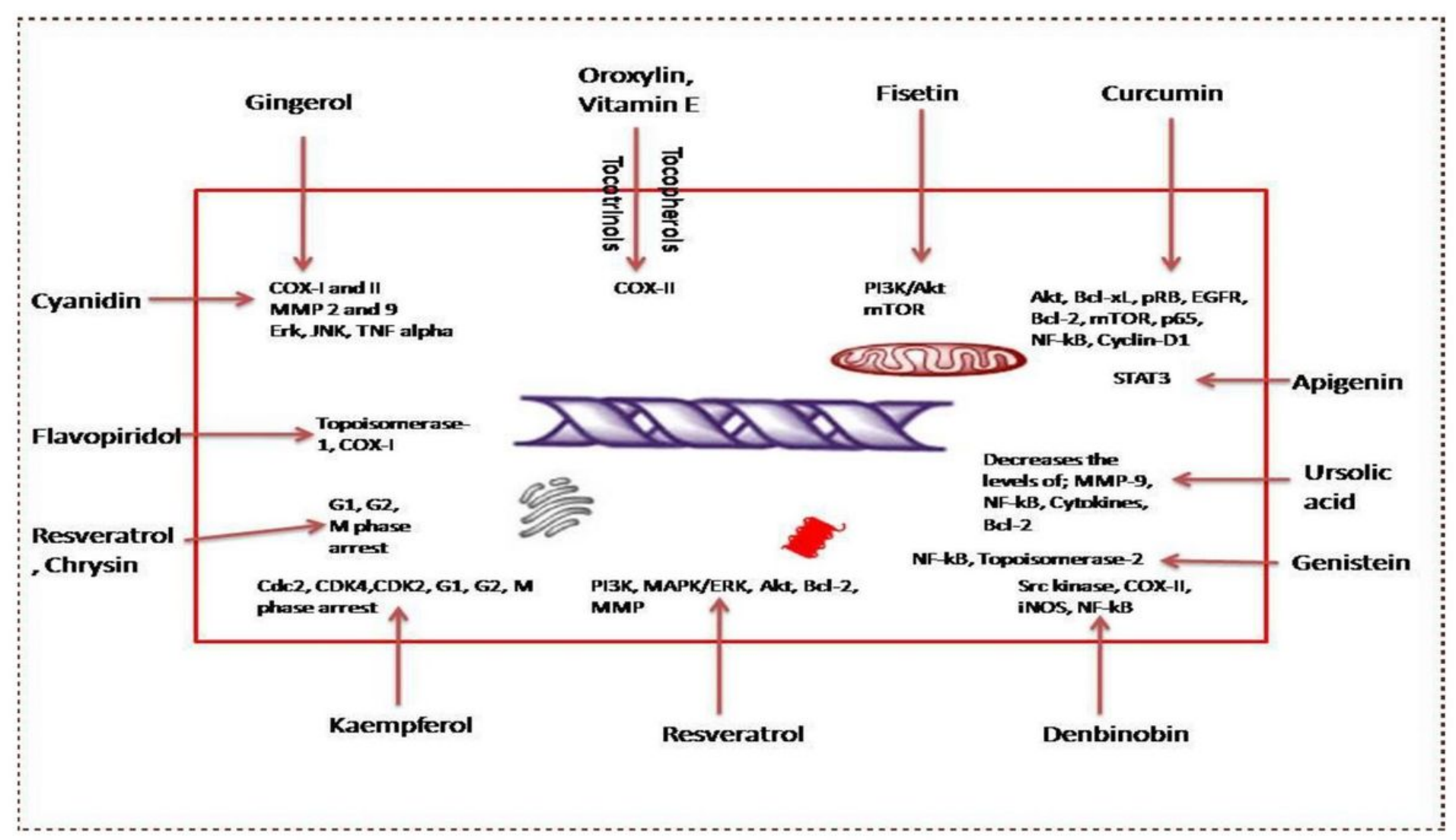

Figure 2

Effect of anticancer plant compounds after activating the onset and progression of cancer by activating the expression of various genes, proteins, enzymes and signaling cascades (after lqbal et al., 2017)

\section{Figure 3}

Effect of anticancer plant compounds after inhibiting the expression of various genes, proteins, enzymes, and signaling cascades and arresting the onset and progression of cancer (after lqbal et al., 2017)

\section{Supplementary Files}

This is a list of supplementary files associated with this preprint. Click to download.

- TABLE.docx 